\title{
Concerning the Numerical Solution of Differential Equations
}

\section{By H. P. Decell, Jr., $\nmid$ L. F. Guseman, Jr. $†$ and R. N. Lea $\dagger$}

Introduction. The stability region of a given difference equation, to be used in the numerical solution of a differential equation $x^{\prime}=f(x, t)$, is a function of the step size $h$ and the partial derivative $\partial f / \partial x$. Higher order methods usually have very small stability regions and thus require very small step sizes. A method for increasing the step size, without violating the stability region constraints, will be developed in the text. The stability region of the difference equation is an interval $[a, b]$ such that, whenever $\bar{h}=h \partial f / \partial x$ lies in the interval, the difference equation yields a stable solution of $x^{\prime}=f(x, t)$ [1], [2]. The size of $\partial f / \partial x$ and $\bar{h}$ dictate the size of $h$.

The Method. Suppose we desire to numerically integrate

$$
x^{\prime}=f(x, t), \quad x\left(t_{0}\right)=a .
$$

Stability considerations depend on $\partial f(x, t) / \partial x$. We show that an alternate differential equation

$$
y^{\prime}=k(y, t), \quad y\left(t_{0}\right)=a
$$

may be developed such that numerical integration of (2) may be more favorable than that of (1) in view of the comparative magnitudes of $\partial f(x, t) / \partial x$ and $\partial k(y, t) / \partial y$, and further, the solution of (1) may be written as

$$
x=\phi[y(t), t] \text {. }
$$

We proceed to determine the functions $\phi$ and $k$. Consider (1) in the form

$$
x^{\prime}=g(x, t)+h(x, t), \quad h(x, t)=f(x, t)-g(x, t)
$$

and suppose that the solution of

$$
z^{\prime}=g(z, t), \quad z\left(t_{0}\right)=a
$$

is known analytically. Let us write this solution in the form

$$
z=\phi(a, t) \text {. }
$$

This function has the property that $\phi\left(a, t_{0}\right)=a$ for all $a$. Let us consider the function $\phi(y(t), t)$. Now

$$
\frac{d \phi(y, t)}{d t}=\frac{\partial \phi(y, t)}{\partial y} \frac{d y}{d t}+\frac{\partial \phi(y, t)}{\partial t}=\frac{\partial \phi(y, t)}{\partial y} \frac{d y}{d t}+g(\phi(y, t), t) .
$$

We determine $y$ so that $x=\phi(y, t)$. Thus

(8) $x^{\prime}=\frac{d \phi(y, t)}{d t}=g(\phi(y, t), t)+h(\phi(y, t), t)=\frac{\partial \phi(y, t)}{\partial y} \frac{d y}{d t}+g(\phi(y, t), t)$

Received October 1, 1965.

$\dagger$ Manned Spacecraft Center, NASA, Houston, Texas. 
by (7) and (4), so that

$$
\frac{d y}{d t}=\left[\frac{\partial \phi(y, t)}{\partial y}\right]^{-1}[f(\phi(y, t), t)-g(\phi(y, t), t)], y\left(t_{0}\right)=a .
$$

The latter boundary condition appears from (1) and the special properties of $\phi$, that is

$$
\phi\left(y\left(t_{0}\right), t_{0}\right)=y\left(t_{0}\right)=x\left(t_{0}\right)=a .
$$

The basic idea in this type of construction is that $\partial y^{\prime} / \partial y$ may be much smaller than $\partial x^{\prime} / \partial x$. This would allow the choice of a larger step size in the numerical solution of Eq. (1) with the given difference equation, without violating the constraints of the stability region. The size of $\partial y^{\prime} / \partial y$ will certainly depend upon the choice of the function $g(x, t)$. It might also be noted that, if $\phi(y, t)$ is a linear function of $y$, then

$$
\frac{\partial y^{\prime}}{\partial y}=\frac{\partial f(x, t)}{\partial x}-\frac{\partial g(x, t)}{\partial x}
$$

It should additionally be noted that, in obtaining from $\phi(y, t)$ the solution of $x^{\prime}=$ $f(x, t), x\left(t_{0}\right)=a$, there is a possibility of amplifying numerical integration errors due to the nature of $\phi(y, t)$.

TABLE 1

$h=.0005$

\begin{tabular}{c|c|c}
\hline$t$ & Direct & Alternate \\
\hline .0000 & 0 & 0 \\
.0080 & 0 & $-.40 \times 10^{-15}$ \\
.4505 & $.10 \times 10^{-13}$ & $-.38 \times 10^{-13}$ \\
.9505 & $.98 \times 10^{-14}$ & $-.97 \times 10^{-13}$ \\
1.4505 & $.99 \times 10^{-14}$ & $-.97 \times 10^{-13}$ \\
1.9505 & $.53 \times 10^{-14}$ & $-.98 \times 10^{-13}$ \\
2.4505 & $.83 \times 10^{-14}$ & $-.97 \times 10^{-13}$ \\
2.9505 & $.12 \times 10^{-13}$ & $-.95 \times 10^{-13}$ \\
3.4505 & $.37 \times 10^{-14}$ & $-.98 \times 10^{-13}$ \\
4.0050 & $.58 \times 10^{-14}$ & $-.93 \times 10^{-13}$ \\
\hline
\end{tabular}

Table 2

$h=.006$

\begin{tabular}{c|c|c}
\hline$t$ & Direct & Alternate \\
\hline .0000 & 0 & 0 \\
.0096 & $-.33 \times 10^{-15}$ & $-.44 \times 10^{-15}$ \\
.5406 & $-.23 \times 10^{-9}$ & $-.62 \times 10^{-14}$ \\
1.1406 & $-.10 \times 10^{-2}$ & $-.90 \times 10^{-13}$ \\
1.6806 & $-.98 \times 10^{3}$ & $-.10 \times 10^{-12}$ \\
2.3406 & $-.20 \times 10^{11}$ & $-.98 \times 10^{-13}$ \\
2.9406 & $-.86 \times 10^{17}$ & $-.10 \times 10^{-12}$ \\
3.5406 & $-.38 \times 10^{24}$ & $-.98 \times 10^{-13}$ \\
4.0206 & $-.77 \times 10^{29}$ & $-.10 \times 10^{-12}$ \\
\hline
\end{tabular}


TABLE 3

$h=.01$

\begin{tabular}{c|c|c}
\hline$t$ & Direct & Alternate \\
\hline .0000 & 0 & 0 \\
.1600 & $.66 \times 10^{-15}$ & $-.95 \times 10^{-16}$ \\
.6500 & $-.15 \times 10^{-9}$ & $-.52 \times 10^{-15}$ \\
1.1500 & $.73 \times 10^{3}$ & $.28 \times 10^{-15}$ \\
1.6500 & $.97 \times 10^{12}$ & $-.17 \times 10^{-13}$ \\
2.1500 & $-.65 \times 10^{22}$ & $-.17 \times 10^{-13}$ \\
2.6500 & $-.51 \times 10^{31}$ & $-.23 \times 10^{-13}$ \\
3.1500 & $-.44 \times 10^{36}$ & $-.22 \times 10^{-13}$ \\
3.6500 & $-.27 \times 10^{36}$ & $-.23 \times 10^{-13}$ \\
4.1500 & $-.16 \times 10^{36}$ & $-.32 \times 10^{-13}$ \\
\hline
\end{tabular}

TABLE 4

$h=.1$

\begin{tabular}{c|c|c}
\hline$t$ & Direct & Alternate \\
\hline .0000 & 0 & 0 \\
1.0000 & $.24 \times 10^{-13}$ & $-.59 \times 10^{-3}$ \\
2.0000 & $.16 \times 10^{-11}$ & $-.73 \times 10^{-3}$ \\
2.4000 & $-.91 \times 10^{-9}$ & $-.736612 \times 10^{-3}$ \\
2.8000 & $.79 \times 10^{-7}$ & $-.736612 \times 10^{-3}$ \\
3.2000 & $.46 \times 10^{-4}$ & $-.736612 \times 10^{-3}$ \\
3.6000 & $-.12 \times 10^{-1}$ & $-.736612 \times 10^{-3}$ \\
4.0000 & $.90 \times 10^{-2}$ & $-.736612 \times 10^{-3}$ \\
4.1000 & $.12 \times 10^{2}$ & $-.736612 \times 10^{-3}$ \\
\hline
\end{tabular}

Note that in Table 4 we begin to see the effects of truncation errors in the alternate solution.

Application. Whenever it is desirable to use higher order methods to control truncation error in the numerical solution of $x^{\prime}=f(x, t), x\left(t_{0}\right)=a$, a small stability region may force the use of an unreasonably small step size. The use of an alternate equation may allow the choice of a larger step size. Consider the differential equation

$$
x^{\prime}=f(x, t)=15 e^{t}-14 x, \quad x(0)=1
$$

whose solution is $x=e^{t}$. Thus, with $g(x, t)=-14 x$, it follows that

$$
\phi(y, t)=y e^{-14 t}
$$

and

$$
\frac{d y}{d t}=15 e^{15 t} ; \quad y(0)=1
$$

In this case, $\partial y^{\prime} / \partial y \equiv 0$ so that the only limitation in choosing $h$ is that of trunca- 
tion errors of the difference method. Moreover, any error introduced in the numerical solution of $y^{\prime}=15 \exp [15 t]$ will be damped by exp $[-14 t]$ in the substitution into $\phi(y, t)=y \exp [-14 t]$.

Tables 1, 2, 3, and 4 give comparisons of relative errors in the numerical solution of $x^{\prime}=15 e^{t}-14 x$ obtained by direct integration, versus the solution obtained by using the alternate equation. The method used is Adams-Bashforth 16th order predictor and Adams-Moulton 15th order corrector. The region of numerical stability (for one application of the corrector) is $-.007 \leqq \bar{h} \leqq .011$. The tables display results using step sizes that caused $\bar{h}$ to lie both inside (Table 1 ) and outside (Tables 2, 3, 4) of the stability region for the direct integration. All integrations connected with the solution using the alternate equation are within the stability region.

Acknowledgment. The authors wish to thank the referees, for their most helpful suggestions and comments, and Mr. John Engvall, for his supporting computer program.

1. R. L. Crane \& R. W. Klopfenstein, "A predictor-corrector algorithm with an increased range of absolute stability," J. Assoc. Comput. Mach., v. 12, 1965, pp. 227-241.

2. L. Fox (Editor), Numerical Solution of Ordinary and Partial Differential Equations, Addison-Wesley, Reading, Mass., 1962, pp. 52-55. MR 26 *4488.

\title{
Some Fourth Order Multipoint Iterative Methods for Solving Equations
}

\author{
By P. Jarratt
}

1. Introduction. Multipoint iterative methods find new approximations to a zero of a function $f(x)$ by sampling $f$ and sometimes its derivatives at each iteration at a number of values of $x$. Although they have not been much used in practice, one interesting class of formulae, investigated by Traub [1, pp. 197-204] is computationally attractive in problems where the evaluation of $f^{\prime}(x)$ is rapid compared with $f(x)$. Such cases arise, for example, when $f(x)$ is defined by an integral. Traub showed that for iterative formulae of the type

$$
\begin{gathered}
x_{n+1}=x_{n}-a_{1} w_{1}\left(x_{n}\right)-a_{2} w_{2}\left(x_{n}\right) \text { where } \\
w_{1}(x)=f(x) / f^{\prime}(x), \quad w_{2}(x)=\frac{f(x)}{f^{\prime}\left[x+\alpha w_{1}(x)\right]},
\end{gathered}
$$

third order processes costing one evaluation of $f(x)$ and two of $f^{\prime}(x)$ per iteration could be constructed by suitable choices of the parameters $a_{1}, a_{2}$ and $\alpha$. It was not possible, however, to obtain fourth order formulae without increasing the number of derivative evaluations. In this paper, a class of iterative methods of the form 